\title{
Mappemonde
}

Revue trimestrielle sur l'image géographique et les formes du territoire

\section{Une régénération urbaine « made in China » : les commerçants chinois et l'expansion des marchés de gros à Budapest}

An urban regeneration "made-in-China": Chinese traders and market expansion in Budapest

Renovación urbana "Made in China": comerciantes chinos y la expansión de los mercados mayoristas en Budapest

\section{Ya-Han Chuang}

Traducteur : François-Michel Le Tourneau

\section{(2) OpenEdition}

Journals

\section{Édition électronique}

URL : http://journals.openedition.org/mappemonde/4141

DOI : $10.4000 /$ mappemonde.4141

ISSN : 1769-7298

\section{Éditeur}

UMR ESPACE

\section{Référence électronique}

Ya-Han Chuang, "Une régénération urbaine « made in China » : les commerçants chinois et l'expansion des marchés de gros à Budapest », Mappemonde [En ligne], 128 | 2020, mis en ligne le 01 juillet 2020, consulté le 02 septembre 2020. URL : http://journals.openedition.org/mappemonde/4141 ; DOI : https://doi.org/10.4000/mappemonde.4141

Ce document a été généré automatiquement le 2 septembre 2020.

\section{c) (1)(2)}

La revue Mappemonde est mise à disposition selon les termes de la Licence Creative Commons Attribution - Pas d'Utilisation Commerciale - Partage dans les Mêmes Conditions 4.0 International. 


\section{Une régénération urbaine « made in China ": les commerçants chinois et l'expansion des marchés de gros à Budapest}

An urban regeneration "made-in-China": Chinese traders and market expansion in Budapest

Renovación urbana "Made in China": comerciantes chinos y la expansión de los mercados mayoristas en Budapest

Ya-Han Chuang

Traduction : François-Michel Le Tourneau

\section{Introduction : les villes post-communistes, une nouvelle approche de la mondialisation et des transformations urbaines}

1 Durant les dernières décennies, le système économique global a connu une transformation spectaculaire marquée par la désindustrialisation des économies d'Europe et d'Amérique du Nord durant les années 1970 et 1980, et par l'émergence de la Chine sur les marchés internationaux depuis les années 1980. L'influence croissance de la main-d'œuvre chinoise bon marché et ses performances économiques globales sont autant de manifestations d'inégalités de développement dans le système économique mondial. Une nouvelle période définie comme «post-industrielle » (Bell, 1973) ou « post-fordiste » (Harvey, 1989a) s'est donc ouverte, caractérisée par la fin de la production standardisée, la notion d'accumulation flexible et la compression des contraintes de temps et d'espace résultant d'un réseau de production toujours plus globalisé. 
2 La production académique sur les conséquences urbaines de la globalisation économique et de la désindustrialisation s'est principalement concentrée sur les transformations du marché immobilier et de la monétarisation des terres dans les économies les plus avancées. Trois approches sont prédominantes.

3 La première, soutenue principalement par la géographie de tradition marxiste, met en avant la néo-libéralisation de la gouvernance urbaine. David Harvey utilise le terme "d'entrepreneuriat urbain » pour décrire les transformations du secteur public, de son rôle de garant du bien-être, via les prestations sociales, à celui d'un catalyseur d'entrepreneuriat (un rôle aussi décrit comme " machine à croissance »), à la poursuite de la croissance économique et de richesses (Molotch, 1976). Ce type de transformation accompagne d'autres processus économiques, en particulier le déclin de l'organisation fordiste du travail, remplacée par la logique de «l'accumulation flexible » (Harvey, 1989a). Ces pratiques particulières ont été intégrées dans la logique du néocapitalisme, la dimension spatiale devenant - à l'égale de celles de classe, de genre et de race - une des dimensions contribuant à la configuration des inégalités sociales (Harvey, 1989b).

4 La seconde approche est celle des global cities, proposée par Saskia Sassen (1994). La sociologue décrit la polarisation de la richesse entre et à l'intérieur des villes. D'une part, le réseau financier mondialisé, ainsi que les flux de capitaux circulant entre certaines villes, divise les villes en les classant entre elles, ce qui les amène à s'opposer les unes aux autres pour attirer plus de capital et devenir plus " globales ». D'autre part, à l'intérieur des villes globalisées, la polarisation à la fois des secteurs participant à l'économie globalisée et de ceux qui fournissent des services " non globaux " s'inscrit aussi dans leur configuration spatiale, créant des inégalités entre les zones où se concentrent les activités économiques globalisées et celles qui sont occupées par des activités moins valorisées.

5 Enfin, la troisième approche attire l'attention sur les villes et les territoires laissés pour compte par la globalisation économique. L'affaiblissement des infrastructures industrielles et la réduction de la stabilité des classes ouvrières posent la question du déclin urbain et de la nécessité de processus de rénovation ou de régénération urbaines (Fol et Cunningham-Sabot, 2010), tout autant qu'elle interroge les processus de gentrification observés dans de plus en plus de villes post-industrielles (Authier et Bidou-Zachariasen, 2008), provoquant des débats autour de la justice spatiale et des normes esthétiques qui inscrivent les distinctions de classe sous la forme de divisions spatiales de l'espace urbain (Zukin, 1993).

6 Les études que nous venons de citer se sont principalement concentrées sur les trajectoires urbaines dans des économies avancées. Le processus de globalisation d'autres secteurs, ainsi que les expériences de villes non fordistes, a peu été documenté. Cet article souhaite étendre la littérature sur le sujet en choisissant un cas différent de conséquences spatiales de la globalisation économique, de plus bas niveau, représenté par la circulation de produits "made in China" dans une ville post-communiste. Comme l'ont indiqué Ferenčuhová et Gentile (2016), l'expérience des transformations urbaines dans les villes post-soviétiques est un sujet sous-étudié, alors que ce type de cas aide à mieux comprendre les conséquences spatiales du développement néo-libéral. Nous nous proposons donc ici d'analyser la création de routes de commercialisation des produits fabriqués en Chine vers l'Europe centrale et de l'Est, et comment la formation de ces routes de commerce de gros - par exemple le commerce inter-entreprises, regroupant la distribution de marchandises depuis l'intermédiaire gérant l'importation 
jusqu'aux clients professionnels qui revendent les produits au consommateur (Pardo et Paché, 2015) - influence les transformations urbaines à l'échelle locale.

7 Notre analyse s'appuie sur des données collectées lors de deux sessions de travail de terrain, un séjour d'un mois à Budapest en octobre 2017 pour visiter les marchés et interroger des commerçants chinois de différentes générations et un second séjour de deux semaines en juin 2019 pour compléter la collecte de données et effectuer un entretien avec le gouverneur d'un district local. Après avoir présenté le contexte de l'arrivée des commerçants chinois à Budapest, nous montrerons que le processus de transformation urbaine s'y déroule selon trois étapes.

\section{Budapest : d'une capitale industrielle à un hub marchand pour l'Europe centrale et orientale}

8 La ville moderne de Budapest a été fondée en 1853, par la fusion de trois villes situées de chaque côté du Danube : Buda, Pest et Obuda. Ainsi que Tosics l'a souligné, la plus grande différence entre les villes communistes et les villes non-communistes repose sur leurs modèles de développement. À la différence des villes des pays capitalistes, les cités communistes sont beaucoup plus influencées par un urbanisme reposant sur une vision politique. Par conséquent, la division entre centre-ville et banlieues, fréquente dans les villes capitalistes, ne structure pas les villes communistes, qui peuvent inclure des zones industrielles jusque dans leurs centres (Tosics, 2006). Budapest est une parfaite illustration de ce modèle. Au nom du développement économique, elle est devenue entre 1950 et 1970 l'une des villes les plus industrialisées de l'Europe centrale et de l'Est. En 1985, la superficie du parc industriel représentait environ 4600 hectares, soit 8,6\% de l'ensemble du territoire urbain (Kiss, 2007). La plupart des sites industriels sont concentrés sur la rive côté Pest du Danube (ibid.).

9 L'implémentation d'une économie de marché en 1990 a modifié ce paysage. La Hongrie a été un pays pionnier parmi les pays d'Europe de l'Est. En promouvant des réformes économiques, elle a permis en 1972 la mise en place d'entreprises privées en jointventures. La création d'un marché foncier depuis 1989 a accéléré le processus de désindustrialisation. De 1990 à 2004, le nombre total d'emplois industriels a diminué de plus de moitié, tombant de 277851 à 117 017, alors que l'emploi dans les services a crû considérablement, passant de 360953 à 649953 (Kiss, 2007). En conséquence, la surface industrielle en ville a diminué de près de $40 \%$. Les secteurs industriels principalement affectés sont l'exploitation minière, la métallurgie, le textile et certaines branches du secteur des machines-outils (Kiss, 2009).

Les immigrants chinois sont arrivés à Budapest dans ce contexte. En 1989, alors que la Hongrie entrait dans l'économie de marché, elle a également mis en place une nouvelle politique leur ouvrant la possibilité de visas. Au moment des événements de Tiananmen - une mobilisation nationale pour un changement de régime, violemment réprimée par le parti communiste chinois au printemps 1989 - des citoyens chinois de diverses classes sociales, travailleurs ruraux, fonctionnaires ou encore intellectuels, se sont intéressés à la possibilité de quitter la Chine. On estime à 40000 ceux qui sont arrivés en Hongrie entre 1989 et 1992, ce qui décida le gouvernement hongrois à restreindre la délivrance des visas (Szabo, 2009). 
11 En Hongrie, comme dans d'autres pays post-communistes dans lesquels les produits les plus usuels n'étaient alors pas toujours disponibles, les produits bon marché venus de Chine répondaient à un besoin urgent et étaient pour cela populaires. Ceux qui les importaient et les vendaient rencontrèrent donc un franc succès commercial. Des routes d'échange émergèrent, tandis que des migrants ruraux (principalement ceux des provinces de Zhejiang et Fujian) créaient un réseau de « marchés chinois » dans toute la Russie. Depuis la capitale hongroise jusqu'aux villages reculés, des vendeurs chinois sont ainsi devenus des intermédiaires permettant aux consommateurs d'accéder à des produits domestiques issus de Chine (Nyiri, 2011). L'expansion de la communauté des grossistes chinois en Hongrie a aussi conduit certains d'entre eux à se délocaliser vers d'autres pays d'Europe de l'Est en raison de la saturation de leur marché de niche ou bien parce qu'ils étaient déçus de la politique fiscale arbitraire du pays ${ }^{1}$. Quelques entrepreneurs ayant réussi ont aussi cherché à établir des succursales dans des marchés émergents comme la Serbie ou la Bosnie-Herzégovine.

L'entrée de la Chine dans l'Organisation mondiale du commerce en 2001 a rendu l'importation des marchandises chinoises bien moins chère qu'elle ne l'était auparavant. Le recours à des intermédiaires pour le commerce transfrontalier est devenu moins important. Certains grossistes ont établi leurs propres usines en Chine et exporté leurs biens directement vers l'Europe. D'autres acteurs dépendent de contacts sur place. Un autre développement important a été l'émergence de l'Italie comme atelier de l'Europe. Alors que le coût de la main d'œuvre en Chine et celui du transport augmentaient, les communautés chinoises de Prato - qui ont revitalisé cette ville italienne, un ancien centre textile, depuis les années 1990 (Ceccagano, 2017) - sont devenues une alternative pour les commerçants implantés en Europe. Plusieurs de nos informateurs en Hongrie ont indiqué commander des marchandises en Italie. Le label « made in Italy » est ainsi omniprésent aujourd'hui chez les grossistes.

Cette brève revue de l'arrivée des immigrants chinois en Europe depuis 1978 montre comment ces derniers ont changé le secteur de l'industrie légère. Dans la partie suivante, nous nous focaliserons sur la manière dont l'expansion du marché chinois a affecté les changements urbains à Budapest. Nous nous intéresserons particulièrement à la façon dont le développement du marché, créé en réponse aux besoins des consommateurs d'Europe centrale et de l'Est, est allé de pair avec la décollectivisation des infrastructures industrielles, transformant les anciennes usines en showrooms et en marchés de marchandises exotiques.

\section{Rénover les friches industrielles: l'expansion des marchés chinois de gros en trois étapes}

14 Les marchés chinois de Budapest se situent dans les $8^{\text {ème }}$ et $10^{\text {ème }}$ districts, dans une vaste zone qui a longtemps été occupée par des usines. Lors du passage du communisme à l'économie de marché en 1989, la ville de Budapest s'est trouvée confrontée au problème posé par la perte de population et par la réduction du tissu urbain. Elle cherche encore des stratégies pour y faire face (Beliczay, 2009). Cependant, les deux districts que nous considérons ont des rôles et des stratégies différentes dans la régénération urbaine. Le $8^{\text {ème }}$ district, Józsefváros, est une zone qui a longtemps souffert d'une mauvaise image à cause de ses habitations collectives dégradées et d'une concentration de population pauvre comprenant une large proportion d'immigrants ou 
d'ouvriers (Czirfusz et al., 2015). Il a fait l'objet d'un processus de rénovation urbaine et de gentrification, tant à cause de son image de «Harlem local» que du fait de sa proximité avec le centre-ville, deux facteurs qui attirent investisseurs et autres tentatives pour réduire le déficit en termes d'offre à loyers intermédiaires (Czirfusz et al., 2015). De son côté, le $10^{\text {ème }}$ district, Kobanya, est beaucoup moins affecté par ce type de projets, car il est situé plus loin du centre-ville. Il se situe dans une zone intermédiaire de Budapest, entre la périphérie et le centre, et il a longtemps été un quartier industriel, préservant encore fièrement cette identité jusqu'à aujourd'hui. D'ailleurs, les impôts sur les activités productives représentent encore la plus grande partie des recettes locales ${ }^{2}$.

Depuis 1989, trois marchés chinois s'y sont installés : "Four Tigers Market » et «Eurosquare » dans le $8^{\text {ème }}$ district, et «Monori Center » dans le $10^{\text {ème }}$. L'évolution de ces marchés révèle les différentes étapes dans le développement des communautés migrantes, mais aussi dans les relations entre ces communautés et les autorités locales, et leurs réponses stratégiques à d'autres acteurs qui entrent en compétition avec elles dans ces mêmes quartiers. On trouve là des éléments significatifs pour la compréhension des processus de compétition foncière et de requalification urbaine dans différents quartiers de Budapest.

\section{“Four Tigers Market" dans les anciens ateliers Ganz}

Figure 1. Reconversion postsocialiste dans le quartier de Józsefváros à Budapest

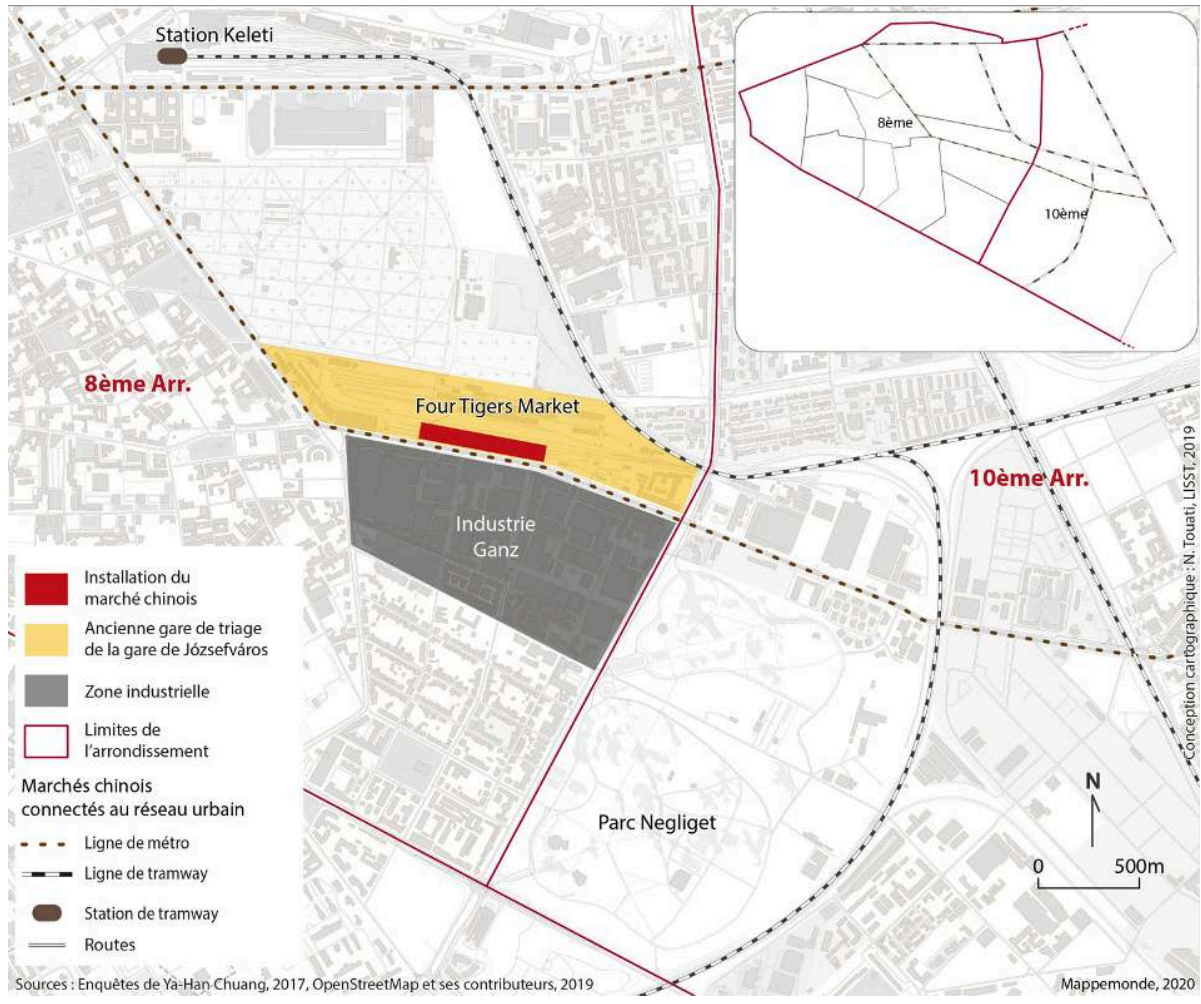

Des marchés chinois dans une friche industrielle (1993-2000).

La première étape de cette transformation urbaine a été la privatisation des anciens Ateliers Ganz, qui appartenaient à la compagnie ferroviaire nationale hongroise 
(figure 1). Ceux-ci ont été transformés en un marché, le "Four Tigers Market» (Józsefváros Kinai Piac en hongrois), inauguré en 1994. Les Ateliers Ganz avaient été fondés en 1844 et contrôlés par une série d'investisseurs étrangers avant d'être nationalisés un siècle plus tard. Le changement de régime en 1989 entraîna une privatisation de l'entreprise, qui développa des joint-ventures avec des investisseurs internationaux à partir de 1991. Une grande partie du patrimoine foncier et immobilier de la compagnie a alors été cédé, puis une série de licenciements d'ouvriers a débuté à partir de $1995^{3}$.

17 Avant 1993, la plupart des marchands chinois circulaient dans différentes zones de la ville où ils écoulaient des vêtements bon marché, souvent de manière illégale, montant par exemple des magasins de chaussures dans des passages souterrains ou sous des ponts. Des marchands plus importants étaient établis dans des marchés plus formels,

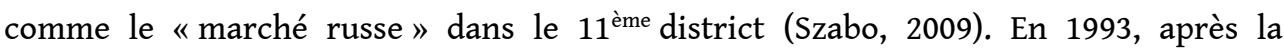
fermeture de plusieurs marchés en périphérie de Budapest, une société immobilière prit les anciennes usines des Ateliers Ganz en location et construisit le Four Tigers Market. Un nombre croissant de grossistes chinois s'installèrent dans la zone, aux côtés d'autres nationalités (Vietnamiens, Turcs, Égyptiens, Ukrainiens, Roumains, etc.). Pour souligner la présence des négociants asiatiques, le gérant de la zone choisit le nom de Four Tigers Market, en allusion aux « quatre tigres » de la croissance économique en Asie (figure 2) $^{4}$.

Figure 2. L'enseigne du Józsefvárosi Piac (Four Tigers Market) à l'entrée des anciens Ateliers Ganz

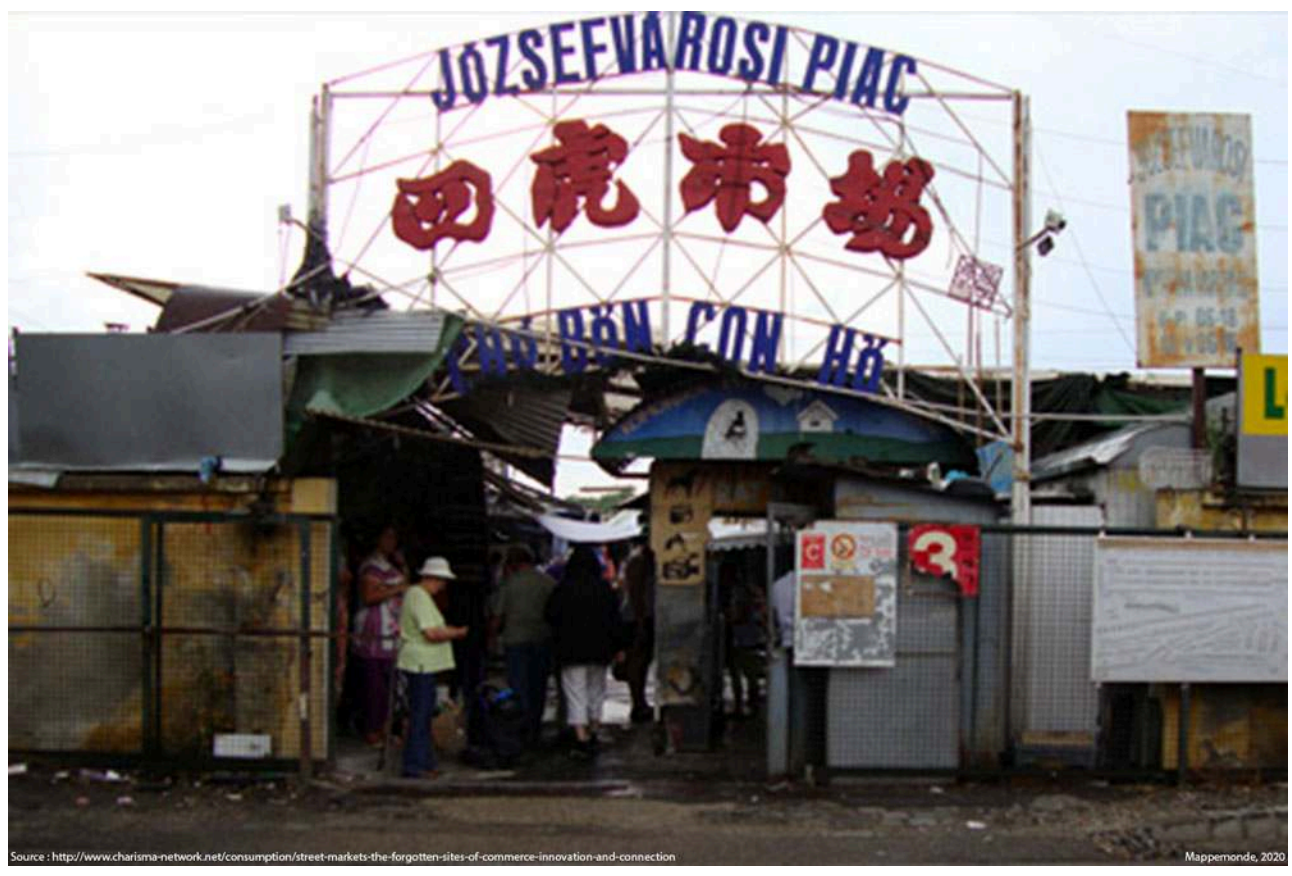

Source : http://www.charisma-network.net/consumption/street-markets-the-forgotten-sites-ofcommerce-innovation-and-connection, page consultée le 4 juin 2018

En tant que première plateforme de circulation de marchandises pour l'ensemble de l'Europe centrale et de l'Est, le Four Tigers Market a attiré des clients de toute la région. De nombreux marchands chinois ont décrit les années 1990 comme un âge d'or. Certains de nos interlocuteurs se souviennent de cette époque comme un moment où "l'argent était comme les grains de sable sur une plage ${ }^{5}$. Les opportunités pour 
s'enrichir dans un marché où régnait alors un capitalisme primitif ont attiré des marchands non seulement depuis la Chine, mais aussi des Chinois installés à l'étranger. C'est ainsi le parcours de $\mathrm{M}^{\mathrm{me}} \mathrm{Lin}$, propriétaire de supermarchés et d'une entreprise spécialisée dans le commerce alimentaire asiatique en Hongrie. Née dans le Qingtian, $\mathrm{M}^{\mathrm{me}}$ Lin possédait plusieurs supermarchés à Brooklyn, New York. En 1995, elle vint à Budapest rendre visite à un ami, lui aussi originaire du Qingtian. Selon elle, elle découvrit qu'il gagnait autant en une seule journée qu'elle en une semaine à New York. Elle décida donc de se lancer dans l'import-export en commandant deux containers de prêt-à-porter en Chine pour les vendre au Four Tigers Market. Cependant, cette première tentative ne fut pas couronnée de succès. Mal informée du marché du prêt-à-porter, $\mathrm{M}^{\mathrm{me}}$ Lin fut en effet trompée par ses fournisseurs chinois qui lui vendirent des produits de mauvaise qualité à un prix très élevé. Après des mois de difficultés, elle était au bord de la faillite. Mais un investissement dans le secteur qu'elle connaissait le mieux l'industrie alimentaire - fit qu'elle put finalement faire des bénéfices et bâtir le plus grand réseau de supermarchés franchisés spécialisés dans le commerce alimentaire asiatique en Hongrie. Aujourd'hui, ses affaires se sont développées dans plusieurs pays d'Europe centrale et de l'Est, de l'Autriche à la Roumanie.

19 Le développement de routes commerciales entre la Chine et l'Europe a aussi placé Budapest sur le chemin des articles de contrefaçon. Comme la Hongrie se trouve au centre de l'Europe, le réseau d'entrepreneurs-migrants du Zhejiang permet aussi aux produits de luxe contrefaits de circuler via le réseau de la diaspora. Un propriétaire de restaurant du Qingtian qui s'est installé à Budapest en 1987 indiquait ainsi : «À cette époque, les membres de nos familles venaient d'Italie ou d'Espagne à Budapest et remplissaient leurs voitures de contrefaçons qu'ils ramenaient chez eux ». Entre-temps, comme dans le cas de la "route des jeans» entre Yiwu et Le Caire (Pliez, 2007), les marchands chinois en Europe constituèrent également leur « route des Nike » à travers l'Europe centrale. M. Wan, le PDG d'une entreprise de chaussures à Budapest, décrit la banalité de la contrefaçon à cette époque : «À cette époque, beaucoup sont venus nous demander de mettre des logos de marques connues sur leurs produits. Beaucoup de mes amis l'ont fait. Moi je voulais créer ma propre marque et j'ai résisté à la tentation, mais beaucoup de mes connaissances pensent que c'était irrationnel de ma part. ${ }^{6}$ Toutefois, si ce type de pratique, typique des économiques informelles, explique l'accumulation rapide de profits, il a également eu pour conséquence un contrôle policier plus intense et des pressions pour la fermeture du marché.

Alors que le commerce de gros de produits «made in China » créait un influx important de marchandises, de travailleurs et de consommateurs, l'ensemble du district de Józsefváros fut aussi l'objet d'un processus de gentrification, dont l'objectif était d'attirer plus de capitaux étrangers et de dynamiser le marché de l'immobilier. Le processus a été lancé par le gouvernement du district, qui souhaitait se débarrasser de l'image de «Harlem local » associée au quartier (Scott et Sohn, 2018). L'exemple le plus représentatif de ses efforts est le projet de la promenade Corvin. Situé le long de la limite du centre-ville, ce quartier, composé à cette époque d'immeubles dégradés, est aujourd'hui totalement occupé par un centre commercial, un casino et des ensembles de logements haut de gamme à destination des élites locales et des touristes (Keresztély et Scott, 2012). Des tensions existent, cependant, entre les acteurs de la gentrification et ceux du marché. La concentration de marchands étrangers et de travailleurs immigrés dans le marché tend en effet à renforcer la stigmatisation de ce district comme 
"ghetto ", ce qui est de moins en moins compatible avec la nouvelle image que veulent donner les développeurs. Depuis le milieu des années 2000, le gouvernement du $8^{\text {ème }}$ district a fréquemment affiché son intention de fermer le marché. En 2014, c'est le gouvernement national qui a annoncé qu'il serait détruit pour faire place à un stade de football ${ }^{7}$.

Ces pressions pour la fermeture du marché ont amené les entrepreneurs chinois à chercher une autre implantation. Deux autres espaces ont alors surgi dans des zones à proximité : « Eurosquare » et le « Monori center ».

\section{Eurosquare : comment des immigrants-entrepreneurs ont transformé les usines des anciens Ateliers Ganz en showrooms}

La seconde étape de l'expansion des marchés chinois a été caractérisée par l'émergence des entrepreneurs chinois comme acteurs du marché immobilier (figure 3). L'expansion du «Four Tigers Market » a encouragé des grossistes à investir dans des immeubles vides de l'entreprise Ganz situés de l'autre côté de l'avenue. Ces investisseurs sont ensuite devenus les acteurs principaux d'un nouveau marché, aujourd'hui appelé « Eurosquare».

Figure 3. Reconversion postsocialiste dans le quartier de Józsefváros à Budapest

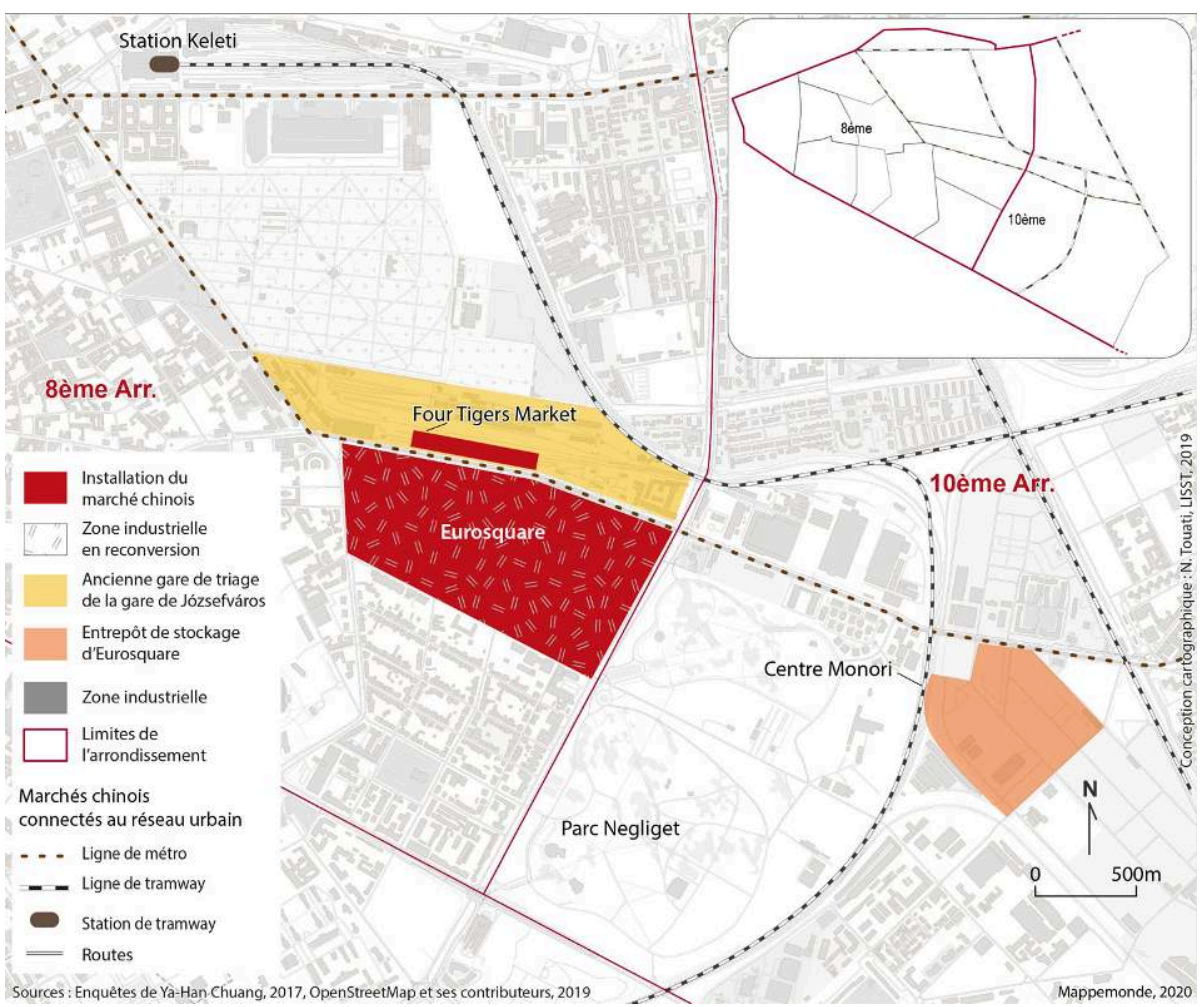

Des zones industrielles aux showrooms organisés (2000-2008)

Depuis 1991, la compagnie Ganz a été transformée en une entreprise semi-publique, attirant un éventail diversifié d'acheteurs pour ses locaux vacants, qui pouvaient facilement être transformés en espaces de stockage. On estime que l'usine et les bureaux Ganz appartiennent aujourd'hui à au moins 140 acheteurs de 20 nationalités différentes. Lorsque la menace d'une fermeture du Four Tigers Market est devenue 
tangible, certains ont eu l'idée d'ouvrir un nouveau marché sur un site proche, non encore reconverti. En 2002, une partie de l'ancienne usine Ganz située au 23-25 avenue Kobanya a été renommée « Eurosquare " (ouya guangchang) et est devenue le deuxième marché chinois de la ville (figures 4 et 5 ).

Figure 4. L'entrée du marché chinois affiche « Bienvenue à Eurosquare »

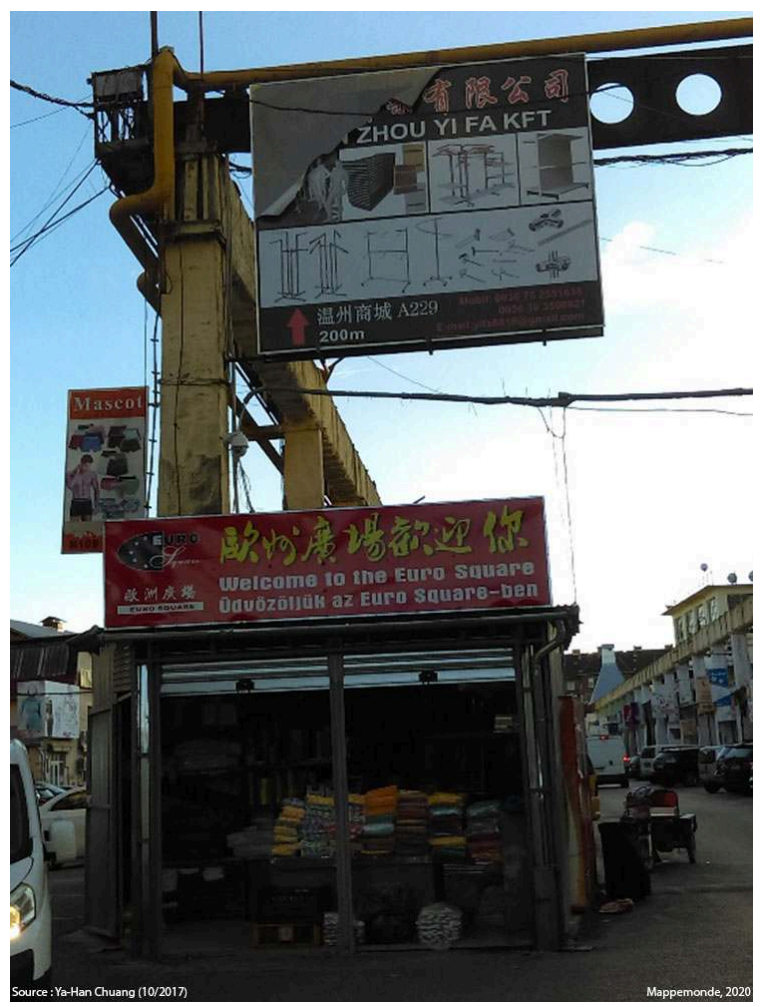

Photographie : Ya-Han Chuang, octobre 2017 
Figure 5. Ancien entrepôt rebaptisé « Avenue du Shanghai »

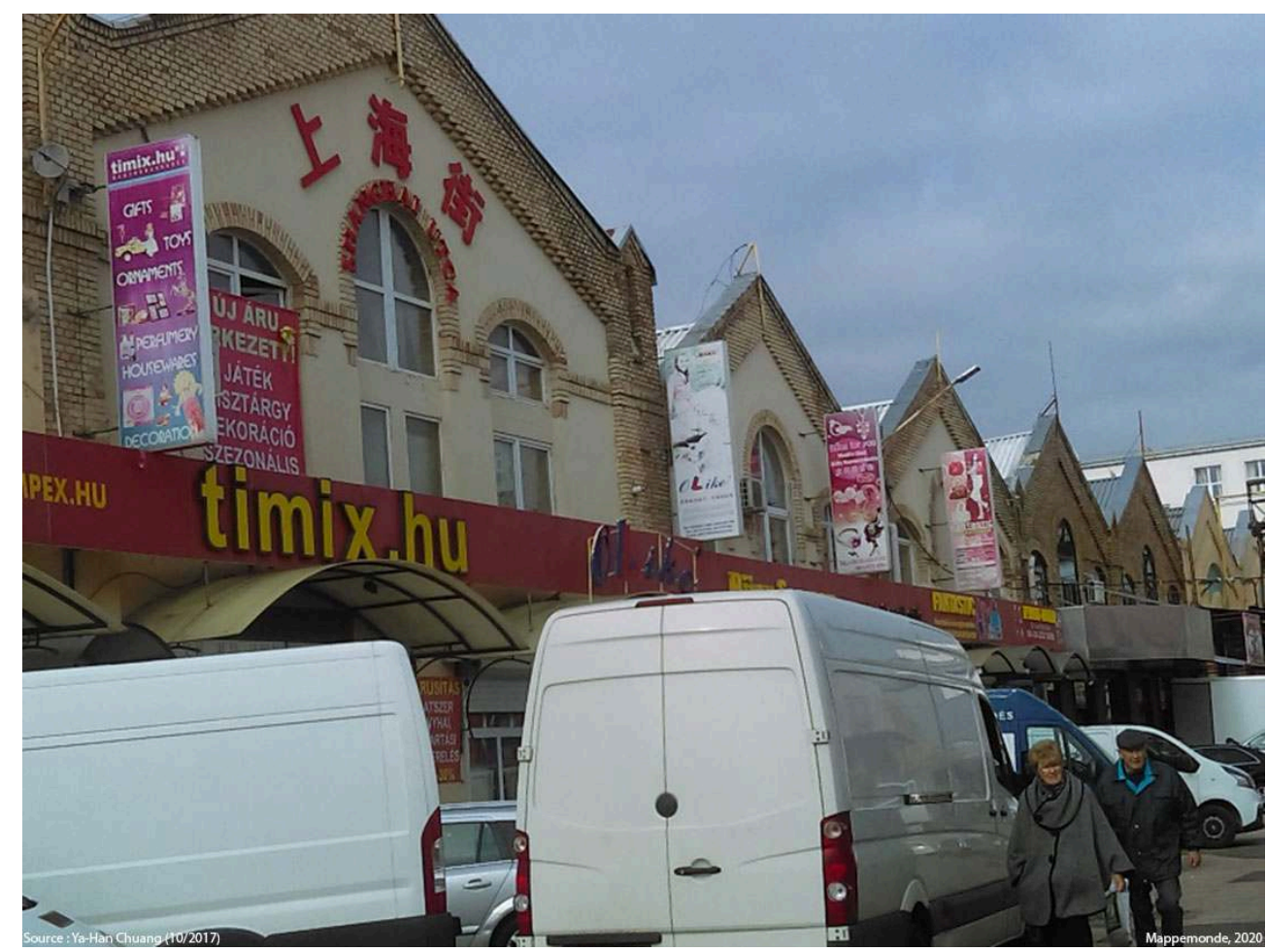

Photographie : Ya-Han Chuang, octobre 2017 patrons de magasin chinois représentaient le groupe le plus important. Cette situation était en grande partie due à l'investissement initial de M. Ye, un homme du Qingtian, arrivé à Budapest en 1989 dès l'ouverture des frontières de la Hongrie. Ancien fonctionnaire, Ye a commencé sa carrière de commerçant à Budapest au Four Tigers Market. Rapidement, le propriétaire de ce dernier lui a délégué une partie du contrat de location et de la communication avec d'autres marchands chinois. À mesure que son entreprise se développait, il put acheter plusieurs édifices industriels sur le site Ganz, en face du Four Tigers Market, qu'il utilisa pour stocker ses marchandises. Progressivement, il subdivisa peu à peu ses espaces et les loua comme entrepôts de gros. Lorsque la pression pour la fermeture du Four Tigers Market s'accrut, un nombre croissant de grossistes chinois s'installa de l'autre côté de la rue à Eurosquare, devenant ainsi les locataires de M. Ye. Nombre d'entre eux sont aussi engagés dans le demi-gros ou le commerce de détail, en particulier ceux qui sont arrivés après 1995 et ceux qui sont entrés sur le marché avec moins de capital de départ.

ceux qui travaillaient au Four Tigers Market, la mémoire des hivers glacials passés à travailler est le souvenir le plus souvent mis en avant pour cet «âge d'or» du commerce. La transition vers Eurosquare a donc représenté pour eux l'accession à de bien meilleures conditions de travail : les espaces d'exposition y sont bien plus grands que les boxes du Four Tigers Market et les locaux peuvent être chauffés ou climatisés selon la saison.

Bien qu'Eurosquare paraisse bien plus organisé, il semble maintenir la même division ethnique du travail que son prédécesseur. Durant notre travail de terrain, accompagnée par un chercheur arabophone, deux commerçants égyptiens ont tenté de 
nous empêcher de prendre des photos de leur magasin de produits électroniques. Dès que la conversation s'est engagée en arabe, ils ont éclaté de rire et nous ont paisiblement expliqué leur réaction. Ils craignaient que nous ne soyons des journalistes venus chercher matière à diaboliser l'Eurosquare market, comme cela s'est souvent produit. Ils nous ont aussi expliqué travailler depuis 25 ans dans ce quartier, et s'être installés juste après la chute du mur de Berlin. En d'autres termes, la fin de la guerre froide a transformé Budapest en point de convergence des routes commerciales venues d'Asie, d'Europe et d'Afrique, attirant des marchands venus du Moyen-Orient et d'Afrique du Nord qui font la navette entre la capitale hongroise et leurs pays d'origine. Janus, un Hongrois employé dans la sécurité du Four Tigers Market, décrit ainsi la situation : «Ici tout le monde a trouvé sa place. Les Chinois font le commerce de gros, les Vietnamiens le commerce de détail, les Arabes (principalement originaires d'Égypte et de Turquie) contrôlent le change. Nous nous sommes beaucoup battus les uns contre les autres dans les années $1990^{8}$, mais maintenant, après toutes ces années, on apprend à vivre ensemble dans le calme $»^{9}$.

Le récit de Janus fait allusion à une division du travail alignée sur les frontières ethnolinguistiques. Selon lui, si Eurosquare est à première vue un hub commercial dominé par les commerçants chinois, on peut aussi le considérer comme un souk musulman (Simone, 2007) comme on peut en trouver à Yiwu (voir l'article d'Olivier Pliez et Said Belgidum à paraître dans le présent dossier). L'arrivée des flux de commerce "made in China » après la fin de la guerre froide a donc contribué à une configuration de « superdiversité » (Vertovec, 2007) dans le contexte du marché.

À l'opposé, le Monori Center, inauguré en 2006, symbolise quant à lui une nouvelle génération de showrooms modernes.

Le Monori Center : rénovation et reconstruction d'un marché de gros dans des locaux industriels privatisés 
Figure 6. Reconversion postsocialiste dans le quartier de Józsefváros à Budapest

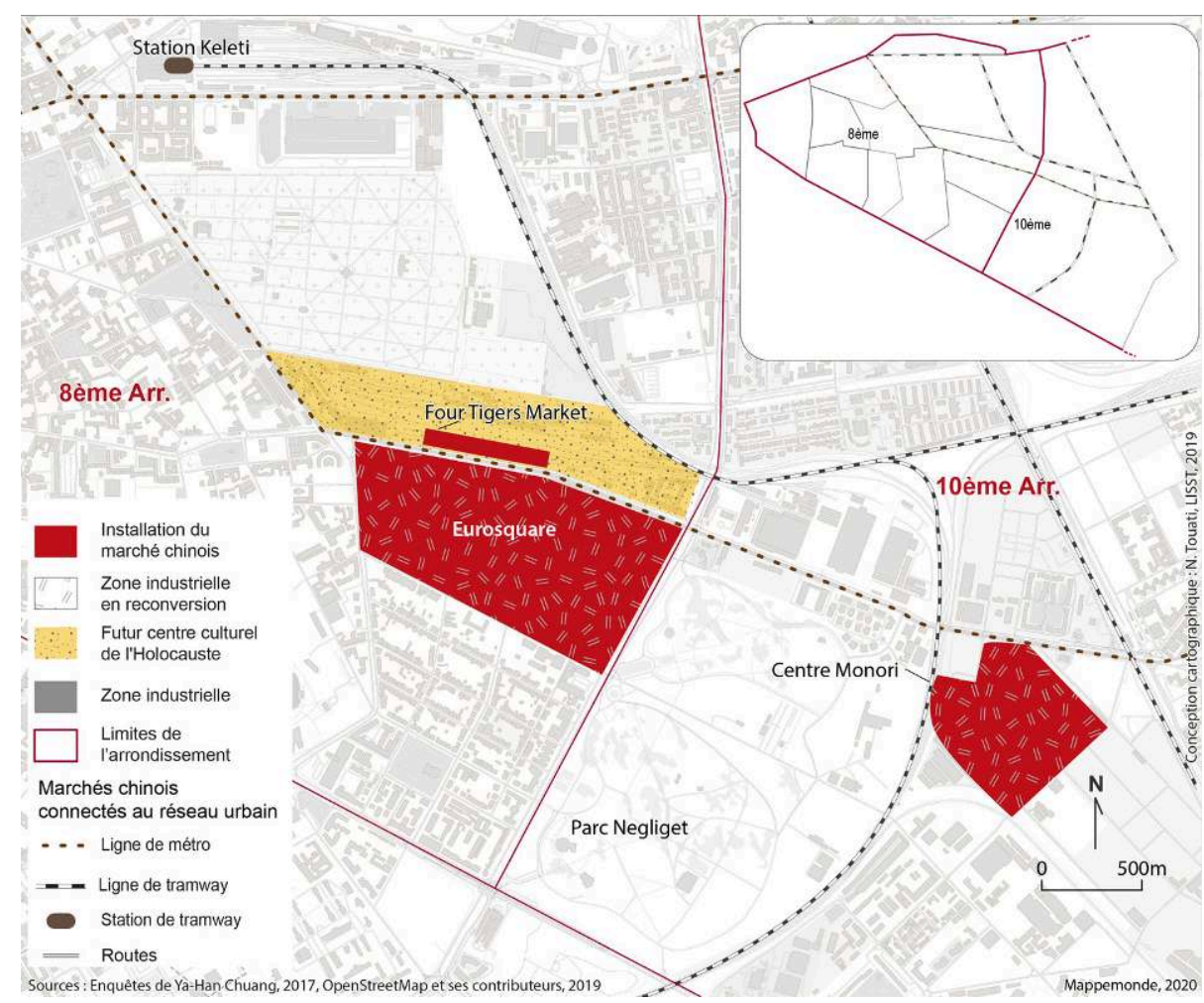

Relocalisation et agrandissement des showrooms chinois de 2008 à aujourd'hui.

La troisième étape de transformations urbaines à Budapest est caractérisée par la rénovation d'immeubles industriels abandonnés, accompagnée de la construction de nouveaux espaces d'exposition ou d'activités commerciales (figure 6). Ainsi est né le Monori Center, un marché de $150000 \mathrm{~m}^{2}$ localisé entre les rues Monori, Szállás et Jegenye, dans le $10^{\text {ème }}$ district de Budapest (Kobanya). Il tire son nom de la rue Monori qui traverse la zone, autrefois occupée par des entrepôts et des usines sous contrôle de l'État. À la fin des années 1990, Song Wanjun, un commerçant du Henan installé au Four Tigers Market, acheta le terrain pour y entreposer son stock. À la différence de Ye Jianxin, le propriétaire d'Eurosquare qui a choisi de préserver la structure des ateliers Ganz, M. Song décida de construire un nouveau bâtiment qui devait refléter l'image de la communauté chinoise de Budapest. Son centre est donc appelé « Tang ren jie » en chinois, ce qui signifie littéralement « Chinatown ». Le propriétaire explique : « Dans de nombreux pays occidentaux, les Chinatowns étaient limitées par le bâti existant. Par conséquent, elles étaient sales et mal organisées. Notre Chinatown a été planifiée et construite sur un espace vierge, elle peut donc se rapprocher des standards occidentaux et être un reflet de la communauté chinoise. " ${ }^{10}$

D'après lui, le marché n'est donc pas seulement un espace de travail pour les marchands chinois; c'est aussi une représentation de la communauté des migrants chinois aux yeux de la population locale. Dans son ambition de construire une Chinatown sur un espace vierge, M. Song a peu à peu transformé les alentours en un réseau de showrooms bien organisés, en rénovant d'anciens bâtiments industriels, ou en construisant de nouveaux espaces d'exposition (figure 7). Ces opérations ne lui permettent pas seulement d'empocher des loyers plus élevés. Elles révèlent aussi son 
intention de construire un marché chinois sur un modèle différent de celui du Four Tigers, qui était mal perçu par la population, en améliorant son aspect esthétique.

Figure 7. D'anciens entrepôts rénovés en face des nouveaux showrooms au Monori Center

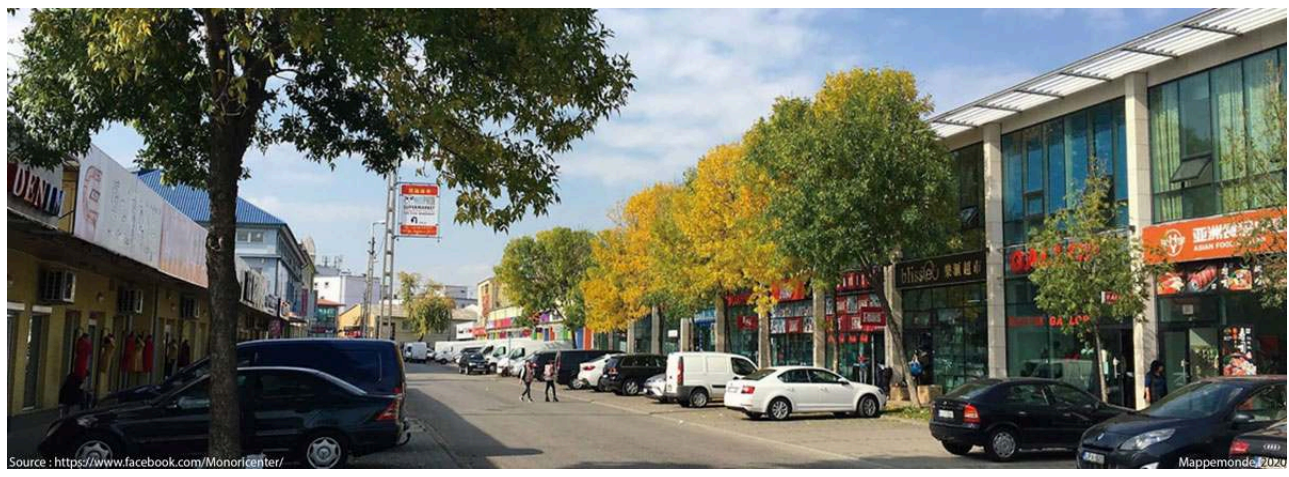

À gauche : bâtiments anciens et usines désaffectées ; à droite : showrooms modernes construits récemment.

Source : page Facebook officielle du Monori Center (https://www.facebook.com/Monoricenter/) consultée le 6 juin 2018

31 Le Monori Center diffère d'Eurosquare sur le plan architectural, mais aussi en ce qui concerne la diversité des commerces. En plus d'une multitude de showrooms spécialisés dans le secteur textile (chaussures, habillement et "mode italienne», c'est-à-dire produite par des entrepreneurs chinois en Italie, équipement de pêche, etc. $)^{11}$, on y trouve également des commerces tournés vers la communauté chinoise: supermarchés, restaurants, bars karaoké, agences de voyages, librairies. Plusieurs institutions communautaires sont installées en périphérie des espaces d'exposition une église, un cabinet d'avocat, une école bilingue, témoignant de la fonction sociale de cette Chinatown discrète.

Au-delà de la satisfaction de la demande des commerçants qui habitent dans le quartier et de celle des résidents chinois des alentours, le choix fait par M. Song résulte aussi d'un facteur structurel. La crise économique de 2008 a sévèrement touché l'économie hongroise, causant une dévaluation du Forint (monnaie locale) de $17 \%$ par rapport à l'Euro et une diminution de $6,7 \%$ du PIB. Les investissements étrangers directs en Hongrie ont diminué de presque $30 \%$ (Egedy, 2012). Du fait de la récession, de nombreux commerçants chinois ont fait faillite et ont choisi de retourner en Chine. Dans ce contexte de crise économique, la demande d'espaces d'exposition s'est trouvée réduite, incitant les constructeurs comme $\mathrm{M}$. Song à innover dans l'usage des espaces commerciaux.

33 Ainsi, l'effort initial de rénovation urbaine impulsé par les activités économiques durant une période de transition postsocialiste permet aujourd'hui de proposer une nouvelle offre culturelle. Durant l'été, de nombreux Hongrois viennent au Monori Center pour profiter du "Monori Night Market», qui est devenu une attraction touristique. Proposant de nombreux restaurants et un grand marché de plein air, la zone du Monori Center représente, à l'inverse de ses prédécesseurs qui étaient des marchés de gros généralement coupés des résidents, une tentative de centre commercial sur le thème des produits exotiques à destination des consommateurs locaux, chinois ou non.

Comme le dit M. Song, "nous voulons construire une vraie Chinatown $»^{12}$. Il projette ainsi de construire un site résidentiel de 200 appartements à proximité. Son intention 
de transformer le Monori Center d'un quartier d'affaires en un quartier qui inclut des fonctions résidentielles est représentative des transformations de l'économie des migrants chinois en Europe. D'un côté, la niche des marchés de gros est presque saturée et la demande pour des espaces d'exposition diminue ${ }^{13}$, ce qui invite à développer de nouvelles stratégies d'usage de l'espace. De l'autre, en 2013, le gouvernement hongrois a annoncé un programme "d'emprunt résidentiel» par lequel des ressortissants de pays non membres de l'UE peuvent obtenir la résidence permanente en Hongrie à condition d'acheter des obligations d'État pour au moins $250000 €$. On estime qu'au moins 4000 citoyens chinois auraient fait cet investissement ${ }^{14}$. Cette nouvelle politique a entraîné un changement qualitatif dans la population chinoise de Budapest, avec une croissance de l'arrivée de migrants de seconde génération. Cette transformation - de migrants économiques (commerçants et travailleurs) à des résidents de classe moyenne supérieure plus aisés et plus transnationaux - est reflétée par la dynamique de l'activité commerciale du Monori Center et par son marché de consommation culturelle.

\section{Épilogue : à qui appartiennent les routes de la soie?}

Notre étude décrit les transformations urbaines à la périphérie du centre-ville de Budapest qui résultent de la formation d'une route de commerce international suite à la chute des régimes communistes en Europe de l'Est. Comme le montre la carte de synthèse (figure 8), au fur et à mesure des années, les flux entrants de marchandises se sont accrus pour approvisionner les marchés de l'Europe centrale et orientale, et les marchés de gros ont cherché des terrains disponibles pour s'étendre, se déplaçant vers la périphérie et adoptant une architecture considérée comme plus compatible avec leur environnement et plus durable pour les propriétaires des magasins. 
Figure 8. Carte de synthèse

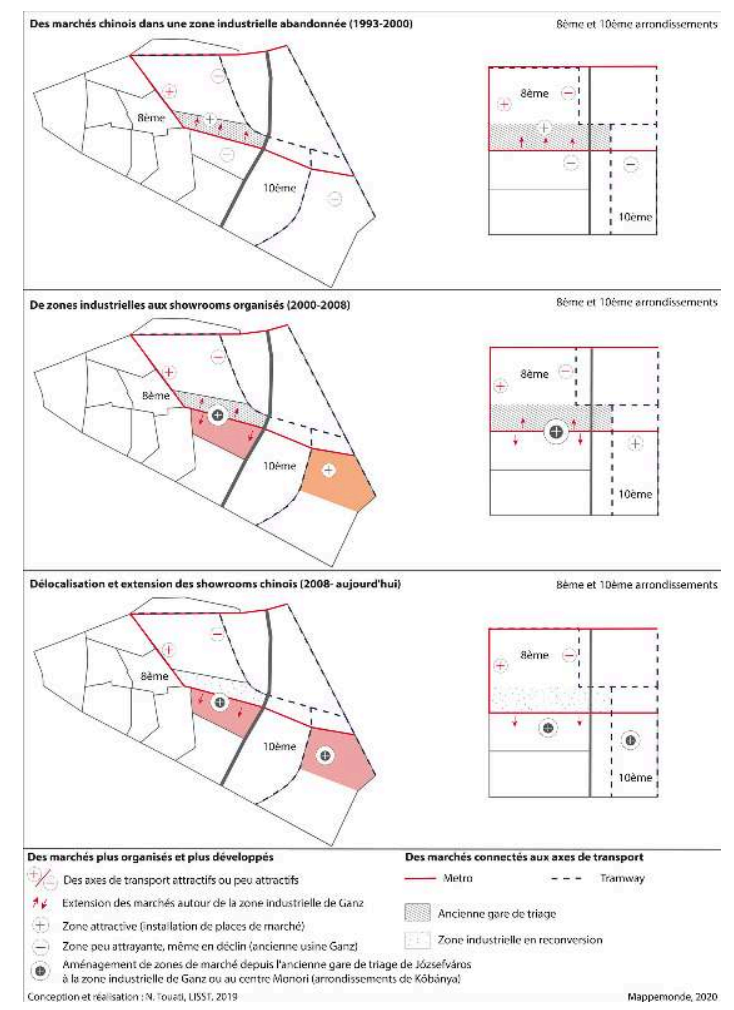

Les différentes étapes de la reconversion postsocialiste dans le quartier de Józsefváros à Budapest.

À la différence du projet de la promenade Corvin, une initiative dirigée par l'État pour attirer des investissements internationaux haut de gamme et développer le secteur du tourisme dans l'ouest du $8^{\text {ème }}$ district de la ville, le développement des marchés de produits "made in China" s'est fondé sur la réponse aux besoins de la population locale. Avec la croissance de la consommation, les commerçants chinois sont passés d'acteurs purement économiques à des acteurs de la régénération urbaine, réutilisant des usines abandonnées et construisant de nouveaux espaces commerciaux et même des bâtiments résidentiels. La dialectique entre les flux de marchandises et de personnes, qui est apparue via la circulation des produits chinois en Europe, a ainsi permis des trajectoires urbaines très différentes et très discrètes, dans le contexte de villes post-communistes manquant de moyens de planification urbaine. Elle révèle aussi une diaspora autonome, constituée de migrants arrivés en Europe centrale et orientale bien avant que l'État chinois ne lance l'initiative des « Nouvelles Routes de la Soie ».

De telles trajectoires collectives, qui représentent des "routes de commerce réelles " (Marsden, 2016), sous-tendues par une globalisation par le bas, contredisent le discours présentant les « Nouvelles Routes de la Soie » comme un projet uniquement tourné vers le développement du commerce. Deux lignes pourraient être poursuivies pour de futures recherches. Sur le plan théorique, notre étude invite à approfondir les liens entre transformation des mobilités des personnes, flux de marchandises et développement dans les années 1990, entre effondrement des régimes communistes et formation du marché commun européen. La région d'Europe centrale et orientale est sans aucun doute stratégique dans ce cadre. Sur le plan empirique, alors que l'État chinois promeut triomphalement son initiative des « Nouvelles Routes de la Soie » dans 
la région, il serait utile d'enquêter auprès des commerçants afin de savoir dans quelle mesure ces projets d'infrastructure modifient leurs pratiques commerciales. Le résultat d'une telle recherche permettrait aux experts non seulement d'apprécier la crédibilité du projet du gouvernement chinois, mais aussi de montrer le rôle respectif de ses dimensions politiques et économiques.

\section{BIBLIOGRAPHIE}

AUTHIER J.-Y., BIDOU-ZACHARIASEN C. (2008). « La gentrification urbaine ». Espaces et sociétés, $\mathrm{n}^{\circ}$ 132-133, p. 13-21.

BELICZAY E. (2009). “Urban regeneration in Budapest”. Study commissioned by the Institute for Transportation \& Development Policy (ITDP).

BELL D. (1973). The Coming of Post-Industrial Society: A Venture in Social Forecasting. New York : Basic Books.

CECCAGNO, A. (2017). City Making and Global Labor Regimes: Chinese Immigrants and Italy's Fast Fashion Industry. Springer.

CZIRFUSZ M., HORVÁTH V., JELINEK C., PÓSFAI Z., SZABÓ L. (2015). “Gentrification and Rescaling Urban Governance in Budapest-Józsefváros”. Intersections. East European Journal of Society and Politics, vol. $1, \mathrm{n}^{\circ} 4$, p. 55-77.

EGEDY T. (2012). "The effects of global economic crisis in Hungary". Hungarian Geographical Bulletin, vol. $61, \mathrm{n}^{\circ} 2$, p. 155-173.

FERENČUHOVÁ S., GENTILE M. (2016). "Introduction. Post-socialist cities and urban theory". Eurasian Geography and Economics, vol. 57, n 4-5, p. 483-496.

FOL S., CUNNINGHAM-SABOT E. C. (2010). « Déclin urbain et Shrinking Cities : Une évaluation critique des approches de la décroissance urbaine ». Annales de géographie, n 674-2010/4, p. 359-383).

HARVEY D. (1989a). The condition of postmodernity: an inquiry into the origins of cultural change. Oxford : Blackwell.

HARVEY D. (1989b). "From managerialism to entrepreneurialism: the transformation in urban governance in late capitalism”. Geografiska Annaler: Series B, Human Geography, vol. 71, nº 1, p. 3-17. KERESZTÉLY K., SCOTT J. W. (2012). “Urban regeneration in the post-socialist context: Budapest and the Search for a Social Dimension”. European Planning Studies, vol. 20, n 7, p. 1111-1134.

KISS É. (2007). “The evolution of industrial areas in Budapest after 1989”. In STANILOV K. (éd.), The Post-Socialist City, Dordrecht : Springer, p. 147-170.

KISS É. (2009). "Major trends in the development of industrial areas of Budapest in the early 21st century". Hungarian geographical Bulletin, vol. 58, n 3, p. 163-180.

MARSDEN M. (2016). “Actually existing silk roads”. Journal of Eurasian Studies, vol. 8, n 1, p. 22-30. 
MOLотсн H. (1976). "The city as a growth machine: Toward a political economy of place". American journal of sociology, vol. 82, $\mathrm{n}^{\circ} 22$, p. 309-332.

NYíRI P. (2011). “Chinese Entrepreneurs in Poor Countries: a Transnational 'Middleman Minority' and its Futures”. Inter-Asia Cultural Studies, vol. 12, n 1, 145-153.

PARDO C., PACHÉ G. (2015). « Introduction générale ». In C. PARDO, éd., Commerce de gros, commerce inter-entreprises : Les enjeux de l'intermédiation, Caen : EMS éditions p. 11-20.

PLIEZ O. (2007). « Des jeans chinois dans les rues du Caire, ou les espaces discrets de la mondialisation ». Mappemonde, $\mathrm{n}^{\circ}$ 88(4-2007). En ligne : https://mappemonde-archive.mgm.fr/ num16/articles/res07404.html

SZABÓ M. (2009). Why Would We Need a Chinatown? The Case of Chinese Entrepreneurs in the Rust Belts of the 8th and 10th Districts of Budapest. Thèse, Budapest : Université d'Europe centrale, Département de sociologie et d'anthropologie sociale.

SASSEN S. (1994). Global city. New York, London, Tokyo. Princeton : Princeton University Press.

SIMONE A. (2007). "The Muslim Street is Everywhere (and soon coming to a theater near you)". Geoforum, vol. 38, n 4, p. 593-596.

scotT J. W., SOHN C. (2018). "Place-making and the Bordering of Urban Space: Interpreting the Emergence of New Neighbourhoods in Berlin and Budapest". European Urban and Regional Studies, vol. $26, \mathrm{n}^{\circ} 3$.

TOSICS I. (2006). "Spatial restructuring in post-socialist Budapest". In TSENKOVA S. ET NEDOVIC-BUDIC Z., dir., The Urban Mosaic of Post-Socialist Europe, Heidelberg : Physica-Verlag, p. 131-150.

VERTOVEC S. (2007). "Super-diversity and its implications". Ethnic and racial studies, vol. 30, $\mathrm{n}^{\circ}$ 6, p. 1024-1054.

XIANG B. (2005). “Transcending Boundaries. Zhejiangcun: the story of a migrant village in Beijing”. Perspectives chinoises, $\mathrm{n}^{\circ}$ 111, p. 91-93.

ZUKIN S. (1993). Landscapes of power: from Detroit to Disney World. Univ of California Press.

ISBN 9780520082885

\section{NOTES}

1. Entretien avec un grossiste en chaussures originaire du Qingtian le 28 octobre 2018.

2. Entretien avec le premier adjoint au maire du $10^{\text {ème }}$ district, le 7 juin 2018, en compagnie de Linda Szabo.

3. Voir le site internet de la compagnie Ganz : http://www.ganz-holding.hu/index.php/en/thepast/198-historical-review, consulté le 5 juin 2018.

4. Entretien avec un ancien employé de la compagnie possédant le marché réalisé le 20 novembre 2017.

5. Entretien avec le manager d'un marché d'alimentation asiatique arrivé à Budapest en 1995. Entretien réalisé le 8 novembre 2017.

6. Entretien avec un responsable de supermarché d'alimentation asiatique arrivé en 1995 à Budapest, $1^{\mathrm{er}}$ mai 2017.

7. Depuis, la zone est restée inoccupée. Le projet de construction du stade ne s'est pas réalisé et certains acteurs du Four Tigers Market considèrent que la décision de fermer le marché provient de la corruption de la classe politique, ou du fait d'une véritable discrimination envers les 
commerçants étrangers (entretien avec un ancien employé du Four Tigers Market, 20 novembre 2017).

8. Il fait ici allusion aux interactions quotidiennes entre groupes ethniques au Four Tigers Market. D'autres informateurs issus de la communauté chinoise ont aussi indiqué que les années 1990 étaient une période d'incidents fréquents et de violence, à la fois à l'intérieur de leur communauté et entre communautés.

9. Entretien du 20 novembre 2017.

10. Source : http://paper.people.com.cn/rmrbhwb/html/2008-10/23/content_123994.htm, page consultée le 06 juin 2018.

11. Liste complète sur le site officiel : http://www.monoricenter.hu/shop/, page consultée le 6 juin 2018.

12. Ibid. note 4 .

13. Plusieurs facteurs structuraux contribuent à ce phénomène, comme la multiplication des marchés de gros dans d'autres villes européennes et la croissance des coûts d'importation depuis la Chine, du fait de l'augmentation des salaires et de l'appréciation de la monnaie chinoise.

14. Le gouvernement hongrois a abandonné ce programme en 2016.

\section{RÉSUMÉS}

Cet article souligne comment l'émergence de routes commerciales entre la Chine et la Hongrie après 1989 a contribué à la transformation urbaine de Budapest. Initiées par des entrepreneurs migrants arrivant dans un nouveau contexte économique, et en réponse à un manque de produits ménagers quotidiens, les pratiques spatiales des commerçants chinois ont évolué en trois étapes : réhabilitation des friches industrielles dans les zones désindustrialisées ; redistribution des droits d'utilisation des terres via une communauté émergente d'acteurs et de locataires ; construction, enfin, d'espaces d'exposition modernes destinés au secteur du commerce de gros. Tout en augmentant la valeur des terrains grâce au développement de l'activité économique et à la gestion immobilière, les entrepreneurs ont également cherché à répondre aux normes esthétiques de la politique de rénovation urbaine. En analysant leurs pratiques spatiales dans une perspective longitudinale de trente ans, l'article contribue à l'étude de la mondialisation économique et de la transformation urbaine à travers un exemple rare de villes postcommunistes.

This article highlights how the emergence of trading routes between China and Hungary after 1989 has contributed to the urban transformation of Budapest. Initiated by migrant entrepreneurs arriving in a new economic context, and in response to a lack of everyday household products, the Chinese traders' spatial practices have evolved through three stages: they started with the rehabilitation of contaminated land within deindustrialized areas; they continued by redistributing the lands' usage rights via an emerging community of property actors and tenants; finally, they constructed modern showrooms dedicated for use by the wholesale sector. While increasing the land value through real-estate operations and management, the entrepreneurs also sought to cope with the aesthetic norms of urban renewal policy. By analyzing their spatial practices through a longitudinal perspective of thirty years, the article contributes to the study of economic globalization and urban transformation via a rare example of post-socialist cities. 
Este artículo destaca cómo, a partir de 1989, la consolidación de la ruta comercial entre China y Hungría contribuyó a la transformación urbanística de Budapest. Iniciada por empresarios chinos, en un contexto económico con carencias en productos básicos para el hogar, ha supuesto prácticas espaciales que han evolucionado en tres etapas: rehabilitación de espacios abandonados en áreas desindustrializadas; redistribución de los derechos de los terrenos mediante una simbiosis de intereses entre propietarios locales y arrendatarios; y, finalmente, la construcción de nuevos espacios para el comercio mayorista. Estas operaciones han supuesto un aumento del precio y de los alquileres de los inmuebles e importantes transformaciones en la política de renovación urbana. A través de tres décadas de prácticas espaciales se pueden estudiar los efectos de la globalización en la transformación urbana de esta ciudad poscomunista.

\section{INDEX}

Thèmes : Les routes de la soie existent déjà. Routes transnationales et places marchandes du made in China entre Asie Afrique et Europe

Mots-clés : diaspora chinoise, Europe centrale et de l'Est, désindustrialisation, rénovation urbaine, commerce de gros

Palabras claves : diáspora china, Europa central y oriental, desindustrialización, renovación urbana, comercio mayorista

Keywords : Chinese diaspora, Central Europe, deindustrialization, urban regeneration, wholesale

\section{AUTEURS}

\section{YA-HAN CHUANG}

Sociologue, post-doctorante, INED 